\title{
Procrastination as a Tool: Exploring Unconventional Components of Academic Success
}

\author{
Damion V. Demeter, Shawn E. Davis \\ School of Professional Psychology, Pacific University, Hillsboro, USA \\ Email: deme0221@pacificu.edu, davissh@pacificu.edu
}

Received March 12 ${ }^{\text {th }}, 2013$; revised April $13^{\text {th }}, 2013$; accepted April $20^{\text {th }}, 2013$

\begin{abstract}
Copyright (C) 2013 Damion V. Demeter, Shawn E. Davis. This is an open access article distributed under the Creative Commons Attribution License, which permits unrestricted use, distribution, and reproduction in any medium, provided the original work is properly cited.
\end{abstract}

\begin{abstract}
Academic procrastination has often been attributed to a fear or avoidance response and elicits negative connotations with both educators and students. Such negative attitudes toward the act of procrastination may result in increased stress for students who procrastinate. However, is this always an appropriate assumption or is procrastination sometimes used as a tool when completing familiar tasks in an advanced educational setting? The current study examines procrastination behaviors of 123 graduate level students currently enrolled across 11 US universities within 20 fields of study. Data collected via self-report questionnaire showed significant relationships between increased academic procrastination and high grade outcomes, when both high levels of familiarity with the testing medium and low levels of fear were present. These data suggest that for settings where the testing medium no longer elicits an acceptable level of fear required for optimal performance, as per the Yerkes-Dodson Law of Arousal, some students may use procrastination to increase arousal. With greater understanding and acceptance of this possibility, students may avoid additional stress associated with non-acceptance of procrastination, which might result in stress levels that are too high and lead to task failure. Additionally, educators who identify this trait in their students may help by creating strategies to aid in this style of task completion.
\end{abstract}

Keywords: Academic Procrastination; PASS; Graduate Education; Yerkes-Dodson

\section{Introduction}

The struggle to avoid procrastination is likely one felt by all students during their academic career. While procrastination may be transitory for many, approximately $70 \%$ of students consistently struggle with academic procrastination (Ellis \& Knaus, 1977) and to some degree the behavior remains universally present (Day, Mensink, \& O’Sullivan, 2000). Persistent procrastination behavior in an academic setting has often been attributed to a deep-rooted fear response (Burka \& Yuen, 2008), a result of perfection seeking (Flett, Blankstein, Hewitt, \& Koledin, 1992), as well as avoidance of an unpleasant task (Solomon \& Rothblum, 1984). Further, researchers have recently identified personality traits (Steel, 2007) and biological factors (Burka \& Yuen, 2008) that can influence and perpetuate this common, and potentially self-defeating, academic behavior. However, is such a global negative interpretation on procrastination behavior oversimplifying a behavior whose impact may vary by population and environment, resulting in a stunted understanding and ability to appropriately mitigate the negative impact? Within the present study, we have provided an alternative interpretation of the causal factors involved in academic procrastination, specifically regarding a graduate level population. We speculate that previous educational experience required for graduate level program admission may result in decreased inherent stress associated with testing mediums. As a result, a percentage of student procrastination may be utilitarian, facilitating optimal performance by increasing arousal levels, as outlined in the Yerkes-Dodson Law of arousal and performance (Yerkes \& Dodson, 1908), rather than existing as a strictly negative behavior.

\section{Traditional Views of Procrastination Causation}

A widely accepted attribution of academic procrastination behavior has been focused on antecedents that hold negative connotations. Solomon and Rothblum (1984), creators of the Procrastination Assessment Scale-Students (PASS), identified two distinct groups based on factors accounting for the highest significant levels of variance within their study. These groups were labeled "fear of failure" and "aversiveness of task" (Solomon \& Rothblum, 1984: p. 508). This, in turn, resulted in the use of these two labels for procrastination in future studies utilizing the PASS to measure procrastination habits. Fear as a cause of procrastination has later been expanded in an exploration of deep-rooted emotional influences resulting from early childhood experiences. Burka and Yuen (2008) hypothesized that early experiences result in the avoidance of uncomfortable feelings that manifest in the academic setting. The authors state that procrastination, in certain contexts, allows the individual to avoid acknowledging their fears, be they a fear of failure, a fear of success, fear of separation, etc. Additionally, Burka and Yuen (2008) explored the neuroscience involved in procrastination, suggesting a hard-wired learned fear response resulting in avoidance, regardless of the actual level of stress imposed by the academic task, points to fear as the basis of all procrastina- 
tion.

Flett et al. (1992) broadened our understanding of procrastination causal factors one step further, expanding it to include perfectionism. The authors' of the study assessed 131 college students that self-identified as procrastinators. The PASS and the Lay Procrastination Scale were utilized to explore procrastination levels. Further, the Multidimensional Perfectionism Scale (MPS) and Burns Perfectionism Scale were included in order to better understand the influence of perfectionism on academic procrastination habits. Flett et al. (1992) observed that while socially prescribed perfectionism was most directly correlated with procrastination, fear of failure was associated with perfectionism. This outcome suggests that while fear of failure is an antecedent of academic procrastination, the behavior is more multidimensional and complex than the direct influence suggested by Solomon and Rothblum (1984).

The influence of perfectionism on procrastination was explored further, focusing solely on a graduate population, by Onwuegbuzie (2000). Onwuegbuzie observed that self-oriented perfectionism and socially prescribed perfectionism were related to procrastination, echoing Flett, et al's (1992) findings, with socially prescribed perfectionism holding a greater influence on procrastination than self-oriented perfectionism. Be it fear of failure, task aversion, or perfectionism, the bulk of commonly accepted attributions of procrastination lie in domains that hold generally negative connotations.

\section{The Yerkes-Dodson Law of Arousal and Performance}

While exploring the relationship between external stimuli and rate of learning in mice, Yerkes and Dodson (1908) observed that as stimulus strength increased, the number of errors declined until a peak was reached and errors began to increase. Hebb's (1955) study on motivation further refined the idea of an optimal level of response and learning, resulting in the inverted-u scale commonly attributed solely to Yerkes and Dodson, stating "there will be an optimal level of arousal for effective behavior" (p. 246). Levels of stimuli that lie before or beyond the optimal level range will be considered mundane or overwhelming, respectively, thus hindering performance.

The inverted-u relationship, as well as the notion of overstimulation resulting in diminished performance not only holds true with practiced learning, but has been found to directly effect memory as well. Deffenbacher, Bornstein, Penrod, and Gorty (2004) observed this relationship while studying the accuracy of eyewitness memory in high stress situations. Their meta-analysis of 27 individual examinations revealed that heightened stress negatively impacted the accuracy of eyewitness identification as well as crime-related details. Further, Deffenbacher, et al. (2004) observed age and individual interpretation of severity of witnessed crime to be influential to the accuracy of recall, reinforcing the necessity of individually varying optimal stress levels for accurate memory retention.

The inverted-learning relationship has also been observed in learning at the physiological level, suggesting its involvement in evolutionary survival. Mateo (2008) explored the relationship of elevated cortisol levels in juvenile ground squirrels and the acquisition of survival-related responses as well as memory retention of the mother's territory. The author concluded that experimentally decreased and increased cortisol levels interfered with the naturally occurring alert response, memory ac- quisition, and memory retention compared to ground squirrels with naturally occurring levels.

\section{A Holistic View of Procrastination}

A number of studies have explored the area of procrastination and, while warning of the commonly experienced negative impacts of such behavior, simultaneously reveal the complexity of its influence on academic behavior. Hancock and Gainey (2004) reject the accepted inverted-u relationship for arousal and performance, stating its simplicity is cause for future study utilizing what they call an extended-u as it is "more congruent with known physiological and psychological effects and also emerging behavioral response data" (p. 13). This work readily acknowledges the variance of individual performance and encourages less simplified and more holistic explanations. Salehi, Cordero, and Sandi's (2010) research focusing on the hippocampus-dependent learning of rats supports the notion of the inverted-u as over simplistic, stating the effect of stress on memory and spatial learning is not uniform in all individuals. Rather, performance in the high and low-stress levels was contingent on the individual's personality-like profile. This research supports the idea that, while a majority of individuals will fall within the expected performance curve, some will continue to experience optimal performance in extreme low and high-stress conditions.

Another atypical view on procrastination is the notion of categorically different types of procrastination based on the effect on the individual or its intended use. Munz, Costello, and Korabik (1975) observed and categorized types and levels of arousal associated with academic test performance. They created 3 levels of arousal consisting of High Activation (H-A), General Activation (G-A), and General Deactivation (G-D). Levels of activation were defined with descriptors such as "jittery", "fearful", and "intense" for H-A, "lively", "energetic", and "peppy" for G-A, and "placid", "calm", or "quiet" for G-D (p. 41). By defining these levels of activation and observing their relation to test performance, Munz et al. (1975) concluded that there were 2 types of arousal, one that enhances and one that impedes performance. Recent research has identified similar categories of procrastination. Chu and Choi (2005) categorized procrastinators as either "passive procrastinators", procrastinators in the traditional sense, or "active procrastinators", those who deliberately suspend their actions for strategic time management. The research of $\mathrm{Chu}$ and Choi suggests that procrastination can be a deliberate action with an expected result rather than a negatively influenced behavior; thus adding support to Solomon and Rothblum's (1984) acknowledgement of procrastination as "not merely a deficit of study habits and organization of time but... a complex interaction of behavioral, cognitive, and affective components" (p. 509).

Ferrari, O'Callaghan, \& Newbegin (2005) also contributed to the categorization of procrastination type and deliberate use of procrastination to facilitate an expected result while examining the difference between "avoidant procrastinators", those whose procrastination stems from fears of failure or success, and "arousal procrastinators", those who procrastinate for a thrill experience (pp. 1-2). Among their sample that included adult participants from three English-speaking nations, $11.5 \%$ selfidentified as chronic arousal procrastinators and 9.9\% selfidentified as chronic avoidant procrastinators (p. 5). By identifying a larger portion of the sample as arousal procrastinators, 
these data directly challenge an accepted view of procrastination that is solely based in understanding the behavior as a response to negatively conceived cognitive and physiological precursors.

The complexity of motivational influences of procrastination, and the categorization thereof, has also been a recent focal point in defining procrastination causal factors. Brownlow and Reasinger (2000) explored the differences between intrinsic and extrinsic motivators' effect on academic behavior, and suggest the combined lack of both intrinsic and extrinsic motivation as the greatest cue that procrastination is likely to occur. Additionally, the authors found that while professors assume that grades are inherent extrinsic motivators, the students in their study who procrastinated reported a lack of sufficient extrinsic motivation as a factor. Thus implying insufficient inherent motivation attributed to the testing medium will necessitate additional, often intrinsically originated, motivational influence that is lacking in some students. However, relying on intrinsic motivation alone cannot reliably predict the avoidance of procrastination, as self-imposed deadlines are first, not as effective as external deadlines and second, many students fail to set selfimposed deadlines optimally (Ariely \& Wertenbroch, 2002).

The collection of work on academic procrastination to date offers a wide range of behavioral, affective, and physiological rationale for the behavior, but scarcely addresses the possible constructive role procrastination may hold for a number of students. Perhaps procrastination in the graduate setting may in fact provide aid rather than hindrance, dependent on a mastery of the necessary skill set (e.g. writing, test taking, and evaluation preparation skills). Yet, given the overwhelmingly negative connotations, those who fall into this population may experience additional distress while procrastinating, thus resulting in stress or activation levels that negatively impact optimal academic performance. The goal of this study is too add additional insight to the complexity of academic procrastination behavior with the hopes of helping academics and students develop a more complete holistic view of the behavior, thus allowing for better mitigation of the possible negative impact.

\section{Method}

\section{Participants}

Participants were recruited via e-mail invitation sent to faculty and department representatives at 11 US universities within 20 varying fields of study. Inclusion criteria consisted only of current enrollment in a graduate-level academic program; both masters as well as doctorate level participants were surveyed. Of the universities contacted, completed surveys were recorded from universities located in the west and south census regions of the United States. Participation was voluntary and incentives were not offered at the study level, however it is unknown to the researchers if professors later offered incentives, such as class credit. Incomplete surveys were excluded from the data set, unless excluded data was confined to the demographic module; discrepancies in demographic and survey sample totals reflect such instances. 123 participants (26 male, 96 female) ranging from $1^{\text {st }}$ year master's to post doctoral academic standing completed the behavioral modules of the survey and those data were included in the final study set. Participant age ranged from 21 to $56(\mathrm{M}=27.7)$ with the majority of participants in the first or second year of doctoral studies (see Table 1).
Table 1.

Demographic information of sample.

\begin{tabular}{|c|c|c|c|c|c|c|}
\hline Characteristic & $\mathrm{N}$ & $\%$ & Mean & SD & Min & Max \\
\hline \multicolumn{7}{|l|}{ Age } \\
\hline Reported & 121 & 98.4 & 27.76 & 6.395 & 21 & 56 \\
\hline Did Not Report & 2 & 1.6 & & & & \\
\hline \multicolumn{7}{|l|}{ Gender } \\
\hline Male & 26 & 21.1 & & & & \\
\hline Female & 96 & 78 & & & & \\
\hline Did Not Report & 1 & 0.8 & & & & \\
\hline \multicolumn{7}{|l|}{ Academic Standing } \\
\hline 1st Year Master's & 12 & 9.8 & & & & \\
\hline 2nd Year Master's & 15 & 12.2 & & & & \\
\hline 1st Year Doctoral & 23 & 18.7 & & & & \\
\hline 2nd Year Doctoral & 28 & 22.8 & & & & \\
\hline 3rd Year Doctoral & 16 & 13 & & & & \\
\hline 4th Year Doctoral & 17 & 13.8 & & & & \\
\hline 5th Year doctoral & 7 & 5.7 & & & & \\
\hline Post-Doctoral & 4 & 3.3 & & & & \\
\hline Did Not Report & 1 & 0.8 & & & & \\
\hline Note. $\mathrm{N}=123$ & & & & & & \\
\hline
\end{tabular}

\section{Measures}

The Procrastination Assessment Scale-Students (PASS), developed by Solomon and Rothblum in 1984, is a two-part assessment that first measures the prevalence of procrastination across 6 academic domains, followed by questions focused on procrastination causal factors. Academic domains addressed in the first section include: writing a term paper, studying for an exam, completing reading assignments, administrative task completion, attending meetings, and a measure of academic task performance in general. Participants are asked to indicate procrastination tendencies in each academic domain on a 5-point Likert Scale ranging from 1 (never procrastinate) to 5 (always procrastinate). Similarly, the degree in which procrastination is a problem for the test taker, the definition of "problem" being open to individual interpretation, in each domain is measured on a 5-point Likert Scale as well, ranging from 1 (not at all a problem) to 5 (always a problem). Finally, participants are asked to what extent they wished to decrease procrastination behavior in each academic domain, again indicating on a 5-point Likert Scale from 1 (do not want to decrease) to 5 (definitely want to decrease).

The second section of the PASS focuses on procrastination reasoning by presenting one procrastination scenario and offering possible rationale for procrastination. Possible reasons include: difficulty in decision making, evaluation anxiety, perfectionism, fear of success, risk-taking, peer influence, rebellion against control, lack of self-confidence, aversion to a task, de- 
pendency and help seeking, lack of assertion, poor time management, and laziness. These items are measured on a 5-point Likert Scale ranging from 1 (not at all reflects why I procrastinated) to 5 (definitely reflects why I procrastinated). Through resulting scores on these two sections of the PASS, researchers can assess: frequency of procrastination, fear of failure, and aversiveness to a task. Procrastination rationale reported which does not fall within the scorable domains, such as laziness, peer influence, and lack of assertion, Solomon and Rothblum suggest are to be addressed when the PASS is administered in a clinical setting in order to begin a conversation about student procrastination influences and later develop individually tailored interventions (Hersen \& Bellack, 1988: p. 360).

In an effort to focus academic procrastination behavior data for this study, 36 questions were created addressing 6 areas of possible academic procrastination: scholastic evaluation tools in general, writing a paper, studying for an exam, weekly reading assignments, academic administrative tasks, and attendance tasks. These questions allowed researchers within the present study to build upon the procrastination reasoning addressed in the PASS by including procrastination behavior covariates. Within each of these 6 areas, participants were asked to indicate on a 5-point Likert Scale ranging from 1 (low or never) to 5 (high or always) the following: procrastination frequency, knowledge level of the subject matter, skill level in the testing medium, fear of the testing medium or process, frequency that procrastination leads to insufficient time allotment, and outcome measured either in grade or completion success.

\section{Procedure}

Participants were directed to an online self-report survey consisting of a brief demographic and academic status module, followed by a procrastination behavior section, consisting of questions addressing fear and knowledge of various testing mediums, procrastination behavior, and academic success. Of the procrastination behavior section's 80 questions, 44 were part of the PASS. Utilization of the PASS provided validity comparison data for questions created by the researchers specifically for this study. The final 36 questions were those created by the authors of this study focusing on procrastination frequency, subject knowledge, testing medium familiarity and fear, task failure rate, and grade outcome. The estimated completion time of the survey was 45 minutes and students were required to complete the survey in one session sans time limit.

\section{Results}

Initially, participant groups were created wherein the type of field the student was from was dichotomized into fields that primarily utilize quantitative testing mediums, such as geography and neuroscience, and fields that primarily utilize subjective testing mediums, such as psychology and sociology. T-tests conducted did not yield any significant differences between male and female participants or between the dichotomized field of study groups. Additionally, an analysis of variance (ANOVA) conducted on academic standing and non-dichotomized field of study were conducted for all main study variables. No significant differences between groups were found. Further, age was not found to be significantly correlated with any study variables; therefore, the following analyses were conducted on the full sample set regardless of demographic consideration.

The PASS was scored for procrastination frequency and fear of failure, strictly adhering to scoring instructions included with the assessment, and these data were compared to study-created question scores for procrastination frequency and fear of the testing medium. Frequency variables were found to be positively correlated, $\mathrm{r}(121)=.709, p<.001$, as were fear variables, $\mathrm{r}(121)=.26, p=.003$. Significant correlations observed between procrastination frequency and procrastination-associated fear measurements suggest convergent validity between the PASS and the study-created questions.

Correlations were conducted between the study-created question categories to explore significant relationships between procrastination frequency, reported grade outcome, and selected academic tasks in an effort to determine the significant relationships involved in academic performance. Procrastination frequency was found to be significantly correlated to overall grade outcome, $\mathrm{r}(121)=.38, p=<.001$, suggesting that, despite frequent procrastination, students successfully completed academic tests regardless of the specific testing medium. This relationship is contrary to the expected relationship between procrastination and grade outcomes based on previous research and suggest a more complex interaction exists.

Further, correlation analysis suggested a difference in types of academic performance and the degree to which performance could be influenced by outside variables. When comparing the relationship between procrastination frequency and academic task completion, academic task completion was not significantly correlated to procrastination, $\mathrm{r}(121)=.005, p=.958$. The difference between procrastination frequency's relationship to academic task completion compared to grade outcomes suggests that while grade outcome is reliant on optimal performance, which can vary in strength based on appropriate levels of activation, academic task completion may be perceived as an all-or-nothing affair. One simply cannot succeed in academic tasks without adhering to attendance and deadline requirements, which are the only way this area is evaluated, therefore the use of procrastination cannot facilitate optimal performance.

Skill in the testing mediums addressed in this survey was negatively correlated with fear of the testing medium $(\mathrm{r}(121)=$ $-.25, p=.005)$ and failure to complete academic tasks ( $(121)=$ $-.21, p=.02)$. This suggests that students' familiarity and practice with testing mediums over time results in a reduced inherent fear response when faced with an academic test, regardless of specific testing medium. Additionally, an increased skill in the testing medium can lead to fewer incidents of insufficient time allocation that possibly contribute to a further reduction of inherent testing medium fear.

A regression analysis was conducted to explore if students' reported procrastination frequency, subject knowledge, skill with the testing medium, fear of the testing medium, and failure to complete academic tasks significantly predicted participants' reported grade outcome. This analysis revealed that procrastination frequency $(\beta=.55, p<.001)$, academic subject knowledge $(\beta=.29, p<.05)$, and fear of the testing medium $(\beta=$ $-.21, p<.05$ ) significantly predicted high grade outcomes (See Table 2). These data suggest that not only does a high level of subject knowledge predict a successful grade outcome, but more surprisingly, low levels of fear and high levels of procrastination frequency do as well. Additionally, of the predictors observed, the most significant predictor of high grade outcomes was procrastination frequency. 
Table 2.

Predictors of self-reported grade outcomes.

\begin{tabular}{ccccccc}
\hline & \multicolumn{5}{c}{ Self-Reported Grade Outcomes } \\
\cline { 2 - 7 } Variable & $\mathrm{B}$ & $\mathrm{SE}(\mathrm{B})$ & $\beta$ & $\mathrm{t}$ & $\mathrm{S}$ Sig. (p) & $95 \% \mathrm{CI}$ \\
\hline Reported Procrastination Frequency & 2.093 & .348 & .550 & 6.022 & $.000^{* *}$ & {$[3.03,11.07]$} \\
Reported Subject Knowledge Level & 1.441 & .606 & .289 & 2.378 & $.019^{*}$ & {$[1.41,2.78]$} \\
Reported Testing Medium Skill Level & .274 & .669 & .051 & .409 & .683 & {$[-1.05,1.60]$} \\
Reported Fear of Testing Medium & -.772 & .301 & -.212 & -2.560 & $.012^{*}$ & {$[-1.37,-.18]$} \\
Reported Failure to Complete Task & -.691 & .407 & -.163 & -1.696 & .093 & {$[-1.50, .12]$} \\
$\mathrm{R}^{2}$ & .331 & & & & & \\
$\mathrm{~F}$ & 13.06 & & & & & \\
\hline
\end{tabular}

Note. $\mathrm{N}=123 .{ }^{*} p<.05,{ }^{* *} \mathrm{P}<.001$.

\section{Discussion}

The goal of this study was to explore the possible existence and utilitarian role of active procrastination (Chu \& Choi, 2005) in a graduate student sample. With alarmingly high levels of procrastination occurring in the academic setting (Ellis \& Knaus, 1977) a holistic view of procrastination behavior is imperative, as a one-size-fits-all outline of causation and mitigation will not foster the greatest levels of student success.

Within the current study, a positive relationship between procrastination frequency and overall grade outcome was found, thus further supporting a dichotomous view of procrastination providing stress that can either enhance or impede performance (Munz et al., 1975). The lack of a significant relationship between academic task completion and procrastination frequency further supports this view, as procrastination was not observed in a domain where performance levels cannot vary and "active procrastination" (Chu \& Choi, 2005) only leads to missed deadlines and failure. Additionally, significant negative correlations between skill in the testing medium and both fear of the testing medium and failure to complete academic tasks were observed. Reported high levels of testing medium skill was expected in a graduate school setting where academic skill levels are assumed to be high, however, low levels of fear and high levels of task completion suggest procrastination causal factors originating somewhere other than fear and avoidance, the sole domains provided by the PASS (Solomon \& Rothblum, 1984). With internal deadlines being less effective than external deadlines (Ariely \& Wertenbroch, 2002), and a lack of appropriate intrinsic and extrinsic motivation typically resulting in procrastination (Brownlow \& Reasinger, 2000), perhaps procrastination is allowing external deadlines to hold more weight and provide a more appropriate level of performance stress for the student to achieve optimal performance. Additionally, regression analysis data revealed procrastination frequency as the strongest predictor of high grade outcomes, providing additional support to theories suggesting utilitarian forms of procrastination (Munz et al., 1975; Chu \& Choi, 2005; Choi \& Moran, 2009; Ferrari, O'Callaghan, \& Newbegin, 2005) as well as the view that the inverted-u relationship for arousal and performance deserves a more holistic explanation (Hancock \& Gainey, 2004) especially in regard to procrastination behavior.

\section{Implications for Students and Educators}

With an understanding of the possible healthy role that pro- crastination may play in some students' academic performance, acceptance of such behavior can mitigate guilt or shame induced stress that can result in academic performance failure resulting from excess stress arousal. Self-shaming and chastising over procrastination should be reduced if such behaviors are thought to act as a tool and do not result in low grades or a failure to complete academic tasks. Educators wishing to preemptively address both the negative as well as possible positive roles that procrastination can have on the individual academic process are encouraged to inform students and encourage them to identify their individual response to procrastination. Have students honestly and objectively quantify their procrastination habits and the effect it has on both grade and project completion outcomes. Emphasize to what level procrastination inhibits students from project completion, and if this threshold is found to be unbroken, attempt to reduce procrastination stigma and added stress. Challenge students who identify procrastination as part of their process to "roll with it" but keep observant of outcomes. Educators may also implement incremental deadlines to help students, especially first year undergraduates, where familiarity and comfort of the testing medium is absent. From there, students can understand procrastination as part of their personal process and develop strategies to either avoid it or work with it within acceptable parameters.

Additionally, a great deal of caution and prudence must be allotted to such procrastination behavior analysis, as the number of individuals this behavior benefits is likely far fewer than those it harms. For many, procrastination is the final variable that contributes to poor grades, underperformance, and even drop out or failure in the academic setting. It is suggested that emphasis falls upon the frequency that procrastination leads to insufficient time allotment as well as grade outcomes when examining if procrastination is part of a student's positive academic process. Once identified, premeditative utilization of procrastination should be discouraged; rather a reduction of shame and guilt paired with understanding and acceptance should be used to reduce excess stress above that which the currently present procrastination provides.

\section{Limitations and Future Direction}

Data collection for this study was conducted primarily during the summer session, which resulted in a smaller sample size than anticipated due to a lower number of programs being in session. Additionally, some programs were at the end of their 
semester, a time when students are less apt to participate in research surveys, instead choosing to focus on finals and semester end projects. Future studies should take this into account, as recruiting during the beginning of the academic semester may yield a larger sample thus allowing for greater generalization.

Efforts were made to collect data from both interpretive areas of study that are evaluated with subjective testing medium, such as term papers, as well as areas of study utilizing a finite answer testing format. Despite this effort, the sample in this study leans heavily toward the social sciences and subjective testing mediums. With a larger sample and more evenly distributed areas of study, researchers could attain not only a better picture of the graduate population as a whole, but would also allow for comparison between testing mediums to explore procrastination's influence on performance for each. Additionally, a prime focus for future research should center on performance and level of procrastination at each graduate year interval to better understand the influence of practice in testing medium familiarity and fear response. Because of the sample size, this study was unable to accurately explore the possible differences in fear and procrastination during each year of graduate studies which would allow for a more holistic view of procrastination causation.

Perhaps the largest possible confounding variables in this study lie in test fatigue and performance inflation. By the time some students arrived toward the end of the 88-questions survey, it is possible that their answers' accuracy may have drifted out of accidental inattention or an effort to quickly complete the survey. Similarly, as is inherent to self-report data collection, academic performance verification was not attained. Despite the anonymous format of the survey, lower than expected observed variance of grade outcomes made researchers question if reported academic performance was inflated by some students in an effort to avoid perceived negative connotations associated with reports of lower scores. Future researchers may identify this kind of reported grade inflation by working with educators to obtain an anonymous report of program grade averages for comparison and analysis.

Future research consisting of larger samples, more areas of academic study, and correcting for possible confounds of this initial study may help both students and educators better understand procrastination behavior and its effects upon academic performance; both potentially positive and negative. While procrastination should not be encouraged, discouragement and stigmatizing of the behavior may not be in all students' best interests either. Only by increasing the understanding of each student's individual performance needs, including where a healthy amount of procrastination may fit into their stress model as a tool, can students reach optimal academic performance without the risk of stress surpassing the individual's threshold, resulting in panic, burnout, or levels of stress that impair performance.

\section{REFERENCES}

Ariely, D., \& Wertenbroch, K. (2002). Procrastination, deadlines, and performance: Self-control by precommitment. Psychological Science,
13, 219-224. doi:10.1111/1467-9280.00441

Brownlow, S., \& Reasinger, R. D. (2000). Putting off until tomorrow what is better done today: Academic procrastination as a function of motivation toward college work. Journal of Social Behavior and Personality, 15, 15-34.

Burka, J. B., \& Yuen, L. M. (2008). Procrastination: Why you do it, what to do about it now (2nd Ed.). Cambridge, MA: Da Capo Lifelong Books.

Chu, A. H. C., \& Choi, J. N. (2005). Rethinking procrastination: Positive effects of "active" procrastination behavior on attitudes and performance. The Journal of Social Psychology, 145, 245-264. doi: 10.3200/SOCP.145.3.245-264

Choi, J. N., \& Moran, S. V. (2009). Why not procrastinate? Development and validation of a new active procrastination scale. The Journal of Social Psychology, 149, 195-211. doi:10.3200/SOCP.149.2.195-212

Day, V., Mensink, D., \& O'Sullivan, M. (2000). Patterns of academic procrastination. Journal of College Reading and Learning, 30.

Deffenbacher, K. A., Hornstein, B. H., Penrod, S. D., \& McGorty, E. K. (2004). A meta-analytic review of the effects of high stress on eyewitness memory. Psychology Faculty Publications, 1.

Ellis, A., \& Knaus, W. J. (1977). Overcoming procrastination. New York: Institute for Rational Living.

Ferrari, J. R., O'Callaghan, J., \& Newbegin, I. (2005). Prevalence of procrastination in the United States, United Kingdom, and Australia: Arousal and avoidance delays among adults. North American Journal of Psychology, 7, 1-6.

Flett, G. L., Blankstein, K. R., Hewitt, P. L., \& Koledin, S. (1992). Components of perfectionism and procrastination in college students. Social Behavior and Personality, 20, 85-94. doi:10.2224/sbp.1992.20.2.85

Hancock, P. A., \& Gainey, N. (2004). From the inverted-u to the extended-u: The evolution of a psychological law. Human Performance in Extreme Environments, 7, 5-14.

Hebb, D. O. (1955). Drives and the c.n.s(conceptual nervous system). Psychological Review, 62, 243-254. doi:10.1037/h0041823

Hersen, M., \& Bellack A. S. (1998) Dictionary of behavioral assessment techniques (pp. 358-360). New York: Pergammon Press.

Mateo, J. M. (2008). Inverted-u shaped relationship between cortisol and learning in ground squirrels. Neurobiology of Learning and Memory, 89, 582-590. doi:10.1016/j.nlm.2007.11.002

Munz, D. C., Costello, C. T., \& Korabik, K. (1975). A further test of the inverted-u hypothesis relating achievement anxiety and academic test performance. The Journal of Psychology, 89, 39-47. doi:10.1080/00223980.1975.9923904

Onwuegbuzie, A. J. (2000). Academic procrastinators and perfectionistic tendencies among graduate students. Journal of Social Behavior \& Personality, 15, 103-109.

Salehi, B., Cordero, M. I., \& Sandi, C. (2010). Learning under stress: The inverted-u-shape function revisited. Cold Spring Harbor Laboratory Press, 17, 522-530.

Solomon, L. J., \& Rothblum, E. D. (1984). Academic procrastination: Frequency and cognitive-behavioral correlates. Journal of Counseling Psychology, 31, 503-509. doi:10.1037/0022-0167.31.4.503

Steel, P. (2007). The nature of procrastination: A meta-analytic and theoretical review of quintessential self-regulatory failure. Psychological Bulletin, 133, 65-94. doi:10.1037/0033-2909.133.1.65

Yerkes, R. M., \& Dodson, J. D. (1908). The relation of strength of stimulus to rapidity of habit-formation. Journal of Comparative Neurology and Psychology, 18, 459-482. doi:10.1002/cne.920180503 